\title{
Desmatamento Recente nos Estados da Amazônia Legal: uma análise da contribuição dos preços agrícolas e das políticas governamentais ${ }^{1}$
}

\author{
Marcelo Dias Paes Ferreira² e Alexandre Bragança Coelho ${ }^{3}$
}

\begin{abstract}
Resumo: O objetivo desse trabalho foi analisar como os preços agrícolas e as políticas públicas afetaram o desmatamento nos estados da Amazônia Legal brasileira no período de 1999 a 2011. Para esse fim, foram utilizados dados estaduais em um modelo de dados em painel e uma metodologia de análise de efeitos líquidos de políticas sobre o desmatamento. Os resultados indicam que o desmatamento está mais relacionado aos preços agrícolas do que às variáveis de políticas associados ao crédito rural e gastos com transporte e agricultura. Entretanto, os resultados parecem indicar que políticas recentes de fiscalização mais focadas, como embargos de comercialização de áreas desmatadas ilegalmente e restrição de crédito para áreas irregulares ou que não cumprem a legislação ambiental, podem ter sido mais efetivas para a redução do desmatamento no estado de Mato Grosso. Sendo assim, concluiu-se que o desmatamento na Amazônia Legal é influenciado tanto pela variação dos preços agrícolas quanto pelas políticas governamentais.
\end{abstract}

Palavras-chaves: Desmatamento, preço da soja, preço do boi, gastos públicos.

Abstract: This paper aimed to analyze the relationship between agricultural prices and government policies related to deforestation within the states of Brazilian Legal Amazon, from 1999 to 2011. We utilized data at state level in a panel data model and a method to measure the net effect of policies over deforestation. Results point out that deforestation in the Brazilian Legal Amazon is more correlated with agricultural prices than government spending on farm credit, agriculture and transportation. Despite this correlation, new command and control policies focused on areas where deforestation were more likely to

1. Este artigo é parte da dissertação de mestrado desenvolvida pelo primeiro autor para a obtenção do título de mestre em Economia Aplicada pela Universidade Federal de Viçosa. Os autores agradecem o apoio financeiro do $\mathrm{CNPq}$ para a realização desse trabalho.

2. Doutorando em Economia Aplicada pela Universidade Federal de Viçosa. E-mail: marcelo_dpf@yahoo.com.br

3. Doutor em Economia Aplicada, professor do Departamento de Economia Rural da Universidade Federal de Viçosa. E-mail: acoelho@ufv.br 
happen were successful, mainly in Mato Grosso State. Finally, we conclude that deforestation is associated with both groups of variables, agriculture prices and policies.

Key-words: Deforestation, soybean prices, cattle prices, government spending.

Classificação JEL: Q23, Q24, Q28.

http://dx.doi.org/10.1590/1234-56781806-9479005301005

\section{Introdução}

O Brasil foi o País que mais desmatou durante as décadas de 1990 e 2000 (FAO, 2010). Boa parte desse processo tem ocorrido na porção do território coberta pela Floresta Amazônica, uma das últimas áreas de fronteira agrícola no mundo (CAMPARI, 2005; HECHT, 1985). Segundo o IBGE (2010), cerca de $20 \%$ da área original da floresta amazônica brasileira já está desmatada. Essa floresta representa cerca de $40 \%$ das florestas úmidas remanescentes no planeta (ANDERSEN et al., 2002) e abriga vida selvagem diversificada, apontada como a área de maior biodiversidade do planeta, o maior banco genético do mundo e detentora de $1 / 5$ da reserva de água potável mundial (IBGE, 2010). Ademais, o desmatamento é responsável por $22 \%$ das emissões brasileiras de Gases do Efeito Estufa (GEE) (BRASIL, 2013), sendo redução do desmatamento mediante intensificação da pecuária um importante mecanismo para reduções nas emissões brasileiras de GEE (COHN et al., 2014; GALFORD, SOARESFILHO e CERRI, 2013).

A porção brasileira da floresta Amazônica, equivalente a cerca de 4 milhões de $\mathrm{km}^{2}$, está inserida na região denominada Amazônia Legal, com território de $5.217 .423 \mathrm{~km}^{2}$, equivalente a $61 \%$ do território nacional. A Amazônia Legal foi criada em 1953 para fins de planejamento político (HOMMA, 2008) e compreende integralmente os estados do Acre, Amapá, Amazonas, Mato Grosso, Pará, Rondônia, Roraima e Tocantins e, parcialmente, o Maranhão (SUDAM, 2010).

O processo do desmatamento na Amazônia Legal está associado a fatores de mercado, tais como variações nos preços das commodities agrícolas, bem como às políticas governamentais que incentivam ou combatem esse problema. Os preços das commodities agrícolas são apontados como causas do desmatamento tanto por trabalhos teóricos (ANGELSEN e KAIMOWITZ, 1999; ANGELSEN, 1999; GEIST e LAMBIN, 2002) quanto por análises empíricas para a Amazônia Legal (ARIMA et al., 2007, 2011; PFAFF, 1999; SILVA, 2009). Assim, constata-se que tais preços possuiriam relação direta com o desmatamento na região. Outros trabalhos indicam que políticas implementadas a partir de 2004 têm contribuído sobremaneira para a redução do desmatamento na Amazônia Legal (ASSUNÇÃO, GANDOUR e ROCHA, 2012, 2013; BARRETO e SILVA, 2010; DE SOUZA, MIZIARA e DE MARCO JUNIOR, 2013; DEFRIES et al., 2013; NEPSTAD et al., 2014). Segundo Barreto e Silva (2010), tais políticas consistiram no aumento da fiscalização, na restrição de crédito rural e de acesso a mercados aos agricultores que não estivessem em conformidade com a legislação ambiental. Tais políticas levaram a uma queda acentuada no desmatamento na Amazônia Legal nos últimos anos ${ }^{4}$.

4. Ver Figura 1 na próxima seção. 
Vários trabalhos foram desenvolvidos a fim de verificar empiricamente o processo de desmatamento na Amazônia Legal. Os principais enfoques identificados na literatura são: desmatamento ligado a questões de mercado ou decisão de investimento (ARIMA et al., 2007, 2011; DINIZ et al., 2009; PFAFF, 1999; PRATES e SERRA, 2009; SILVA, 2009; SOUZA-RODRIGUES, 2012); os que investigam o desmatamento pela ótica dos pequenos produtores (CAMMELLI, 2013; WALKER, MORAN e ANSELIN, 2000); os que associam o desmatamento ao tamanho dos estabelecimentos agrícolas (FÉRES e ARAUJO, 2013; MARCHAND, 2012); os que buscam identificar como as instituições e os direitos de propriedade afetam o desmatamento (ARAUJO et al., 2009; SANT'ANNA e YOUNG, 2010); e os que consideram o papel das políticas públicas como motivador do desmatamento (ASSUNÇÃO, GANDOUR e ROCHA, 2012, 2013; DE SOUZA, MIZIARA e DE MARCO JUNIOR, 2013; DINIZ et al., 2009; PRATES e SERRA, 2009; WEINHOLD e REIS, 2008). Este trabalho pretende complementar os demais tratando três pontos. Primeiramente, fez-se uma abordagem mais ampla dos efeitos de políticas públicas sobre o desmatamento na Amazônia Legal, expandindo para toda região o trabalho realizado por Prates e Serra (2009) para o Pará, que buscou medir os efeitos dos gastos do governo federal em crédito rural, agricultura e transporte sobre o desmatamento. Segundo, utilizou-se uma metodologia de análise do efeito líquido de políticas sobre o desmatamento proposta por Combes Motel, Pirard e Combes (2009), que complementa os estudos de Assunção, Gandour e Rocha (2012, 2013), cujos resultados apontaram que políticas de comando e controle foram efetivas para a redução do desmatamento nos últimos anos. Terceiro, este trabalho procurou verificar se há endogeneidade do desmatamento com crédito rural e população na Amazônia Legal, o que não foi realizado por nenhum dos trabalhos citados. Entende-se, contudo, que o presente trabalho não capta um importante aspecto do desmatamento na Amazônia Legal: a participação da agricultura de subsistência sobre a conversão de áreas de floresta e áreas agrícolas.

Do exposto, esse estudo teve por objetivo geral analisar como os preços das commodities agrícolas e as políticas públicas afetaram o desmatamento nos estados da Amazônia Legal brasileira no período de 1999 a 2011. Especificamente, pretendeu-se: identificar os principais determinantes do desmatamento na Amazônia Legal a nível estadual e verificar se as políticas públicas tiveram efeitos sobre o desmatamento nos estados da Amazônia Legal ao longo do tempo.

Além desta, compõem esse trabalho mais cinco seções: a evolução do desmatamento na Amazônia Legal é analisada na seção 2; na seção 3 , são expostas as teorias das causas indiretas e subjacentes do desmatamento, do desmatamento como decisão de investimento e a abordagem do desmatamento estrutural e observado; a seção 4 diz respeito aos métodos econométricos utilizados e à fonte dos dados; os resultados são apresentados e discutidos na seção 5; e a seção 6 apresenta as considerações finais.

\section{Evolução do desmatamento na Amazônia Legal}

O desmatamento na Amazônia Legal brasileira tem seguido o cenário político e econômico brasileiro. Como se pode observar na Figura 1, a seguir, o desmatamento não seguiu uma tendência bem definida entre o final dos anos 1980 até 2013, mas a queda é consistente a partir de 2005. Em todo o período, a taxa de crescimento do desmatamento foi negativa, apresentando média de $-3,5 \%$ ao ano.

Durante o período de instabilidade econômica no início dos anos 1990, o desmatamento apresentou números relativamente baixos. $\mathrm{O}$ pico no desmatamento observado em 1995 deveu-se à retomada da estabilidade e crescimento do consumo interno após a implantação do Plano Real em 1994 (FEARNSIDE, 2005). Depois desse pico, o governo federal modificou o Código Florestal, determinando que a área de reserva legal pas- 
094 - Desmatamento Recente nos Estados da Amazônia Legal: uma análise da contribuição dos preços agrícolas e das políticas governamentais

Figura 1. Evolução do desmatamento na Amazônia Legal brasileira em km² de 1988 a 2013*

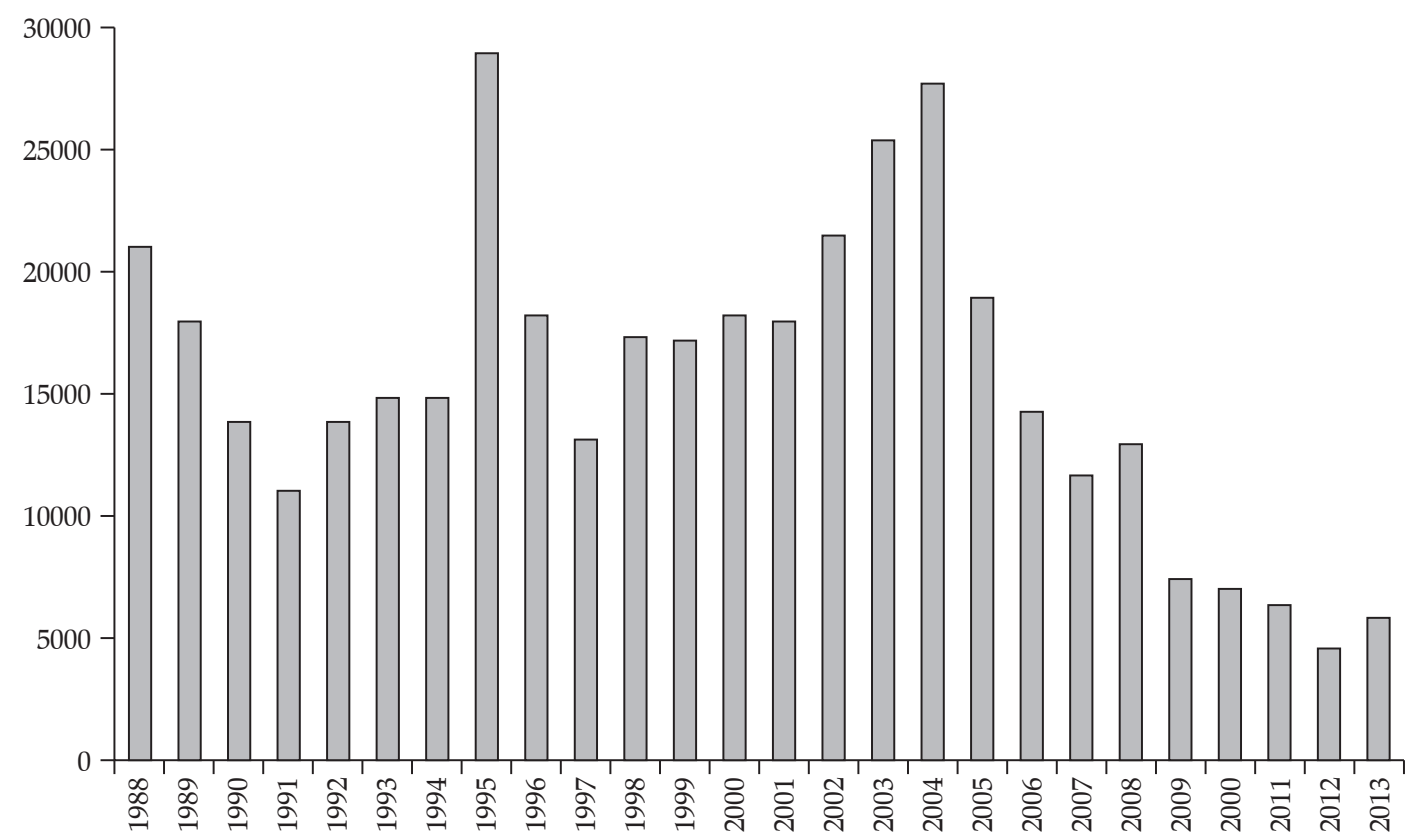

* O desmatamento em 1988 equivale à média do desmatamento da Amazônia Legal de 1977 a 1988; o desmatamento nos anos de 1993 e 1994 é a média entre esses dois anos; e o desmatamento em 2013 representa uma estimativa, enquanto os demais anos são dados consolidados.

Fonte: Inpe (2014).

sasse de 50\% para $80 \%$ na Amazônia Legal, o que pode ter reduzido o desmatamento nos anos seguintes. O desmatamento voltou a crescer de forma mais expressiva a partir de 2002, acompanhando o aumento de preços das commodities agrícolas, especialmente a soja, apresentando outro pico em 2004. A partir de 2005, o preço da soja caiu, reduzindo o desmatamento. Com aplicações de políticas mais consistentes e focadas a partir de 2008, o desmatamento caiu drasticamente (ASSUNÇÃO, GANDOUR e ROCHA, 2012, 2013; BARRETO e SILVA, 2010; DE SOUZA, MIZIARA e DE MARCO JUNIOR, 2013; DEFRIES et al., 2013; NEPSTAD et al., 2014). Por fim, a partir de 2009, o desmatamento na Amazônia Legal foi bem abaixo dos anos anteriores, apresentando

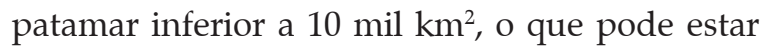
relacionado com as políticas de combate ao desmatamento aplicadas nos últimos anos.

De acordo com Barreto e Silva (2010), essas políticas foram mais abrangentes do que as aplicadas anteriormente. Aqueles autores destacaram três políticas importantes que foram aplicadas a partir de 2008: inspeções de campo mais intensivas e focadas; embargo econômico de áreas ilegalmente desmatadas e restrição de crédito aos agricultores que não cumprissem a legislação ambiental. Na primeira ação, as fiscalizações foram direcionadas aos 36 municípios com maiores índices de desmatamento na Amazônia Legal (do total de 720), responsáveis por $50 \%$ do desmatamento. Iniciado em março de 2008, as ações incluíam a aplicação de multas, confisco de bens e embargos de uso das áreas desmatadas. Como resultado, o número de bens confiscados e de áreas embargadas cresceu 53\% em relação a 2007 nesses 36 municípios, ao passo que, nos demais, esse crescimento foi de $11 \%$. Associado a essa última ação, o embargo de áreas desmatadas ilegalmente incluía a proibição de venda de produtos originários dessas áreas. Esse último instrumento não punia apenas o produtor rural, pois aplicava penalidades aos outros agentes da cadeia que comprassem produtos agrícolas dessas áreas embargadas, sobretudo os frigoríficos. A terceira política consistia na exigência de 
que, a partir de julho de 2008, os proprietários de áreas maiores que 400 hectares comprovassem que tinham o título da terra válido (ou estavam iniciando o processo de obtenção) e que tivessem o licenciamento ambiental para obter crédito agrícola. Dessa forma, essa política restringiu sobremaneira o acesso a crédito para aqueles produtores com maior probabilidade de realizar desmatamento ilegal (BARRETO e SILVA, 2010).

\section{Referencial teórico}

\subsection{Causas do desmatamento}

Angelsen e Kaimowitz (1999) constaram que as variáveis que influenciam o desmatamento se encontram em dois níveis de análise. O primeiro nível de influência é o imediato, diretamente relacionado com as decisões dos agentes econômicos do desmatamento. Entre os parâmetros de decisão desse nível estão compreendidos aqueles pertencentes às dimensões das instituições, infraestrutura, mercados e tecnologia. O segundo nível de influência se dá de modo subjacente ou indireto, relacionado às variáveis no nível macroeconômico e os instrumentos de política. Geist e Lambin (2002) também dividem as causas do desmatamento em imediata e subjacente. As imediatas estão associadas às atividades humanas ou ações imediatas a nível local, como a expansão agrícola relacionada ao uso da terra. Já forças subjacentes são aquelas relacionadas com a dinâmica populacional e a política agrícola.

As causas imediatas do desmatamento são os preços dos produtos agrícolas, os preços dos insumos agrícolas, as rendas não agrícolas da população rural, a disponibilidade de crédito rural, o progresso tecnológico nas fronteiras agrícolas, a acessibilidade e estradas, o regime de direitos de propriedade e os preços da madeira. Como causas subjacentes estão o crescimento da população, o nível de renda, o crescimento econômico, o progresso tecnológico, a dívida externa, a liberalização econômica e a desvalorização cambial (ANGELSEN e KAIMOWITZ, 1999).
Angelsen (1999) argumenta que o desmatamento, como consequência da expansão da fronteira agrícola, tem que ser tratado como uma decisão de investimento, dado que a retirada de floresta, geralmente, gera direitos de propriedade sobre a área desmatada. Dentre os pressupostos teóricos sugeridos por Angelsen (1999), Silva (2009) argumenta que o mais adequado à Amazônia Legal brasileira é o da economia aberta pequena e de acesso livre. Essa abordagem pressupõe que os preços são exógenos, a população é determinada endogenamente, a produção é vendida em mercados e os direitos de propriedade não são definidos, estimulando o desmatamento para adquirir a posse da área.

O desmatamento está positivamente relacionado com as rendas esperadas das atividades agrícolas que, por sua vez, estão associadas aos preços das commodities agrícolas, aos custos agrícolas e às características associadas à cada região. Como características específicas de cada estado, podem ser citadas a distância do estado de um grande mercado de produtos agrícolas, área de estoque florestal, a população, a densidade populacional, a renda absoluta e per capita, características edafoclimáticas etc.

O custo da atividade agrícola é função do custo de limpeza da área a ser desmatada, dos custos da produção agropecuária, das políticas governamentais e das variáveis institucionais. Entre as políticas governamentais estão o acesso ao crédito agrícola subsidiado, a construção de estradas etc. Como variáveis institucionais estão as políticas de fiscalização, prevenção do desmatamento, legislação de direito de propriedade etc. Dessa forma, os agentes econômicos envolvidos no desmatamento maximizam o lucro esperado escolhendo o nível de desmatamento, considerando os preços agrícolas, tendo como restrições as condições de cada estado, os custos agrícolas e de limpeza, as políticas governamentais e o ambiente institucional (SILVA, 2009).

Na ótica do desmatamento como decisão de investimento, podem-se dividir as variáveis em três tipos, quais sejam: condições de mercado, influência política e condições iniciais (SILVA, 
096 - Desmatamento Recente nos Estados da Amazônia Legal: uma análise da contribuição dos preços agrícolas e das políticas governamentais

2009). Dentro do primeiro grupo estão os preços dos produtos agrícolas, e os custos associados ao desmatamento e à atividade agropecuária. Se os preços aumentarem, o desmatamento aumenta. Se ocorrer um aumento nos custos, haveria redução nos níveis de desmatamento. Barreto, Pereira e Arima (2008) sugeriram que o desmatamento na Amazônia Legal estaria associado às mudanças defasadas nos preços agrícolas. Essa relação seria justificada pela incerteza que os produtores rurais têm na rentabilidade corrente da atividade. Assim, eles utilizam as informações de preços da safra anterior para a tomada de decisão presente, sendo essa decisão relacionada ao movimento da fronteira agrícola e ao desmatamento.

Dentro do grupo de políticas estão as variáveis institucionais e as políticas de crédito. As variáveis institucionais podem influenciar o desmatamento de diversas maneiras: espera-se redução no desmatamento, por exemplo, se a fiscalização aumentar, políticas de proteção forem implementadas ou a legislação sobre a posse da terra se tornar mais rígida. Políticas de crédito agrícola subsidiado afetam o desmatamento positivamente, uma vez que relaxam a restrição de capital dos produtores rurais e permitem maior investimento.

Por fim, no terceiro grupo estão as características de cada estado. Espera-se que, quanto maior o crescimento econômico e populacional, maior será o impacto sobre o desmatamento. Assume-se que o desenvolvimento econômico possua uma relação de Kuznets ambiental com o desmatamento, ou seja, à medida que a renda per capita aumenta, o desmatamento também se eleva, atingindo um máximo em determinado nível de renda para depois decrescer, apresentando relação de "U" invertido.

A área inicial de floresta, ou estoque de floresta ao longo do tempo, possui relação positiva com o desmatamento. Isso ocorre por duas razões: primeiro, quanto menor a área de florestas, mais onerosa é a extração de madeira devido às atividades ligadas ao transporte e à escassez de madeira; segundo, quanto menor a área de floresta, maior a disponibilidade de terras para serem utilizadas na agropecuária, diminuindo a pressão por novas áreas.

A área relativa de floresta, percentual de área de floresta remanescente, também está relacionada com o desmatamento, pois pode estar associada ao processo de fragmentação, ou dispersão de pequenas áreas de florestas. Assim, quanto menor a relação floresta/área, maior a probabilidade de haver áreas de florestas fragmentadas. Essa fragmentação torna as florestas mais suscetíveis a queimadas e aumenta a mortalidade das árvores (NEPSTAD et al., 2008).

\subsection{Efeitos líquidos de políticas governamentais sobre o desmatamento}

Buscando relacionar as causas do desmatamento com os esforços de redução, Combes Motel, Pirard e Combes (2009) introduziram a abordagem do desmatamento estrutural. Segundo esses autores, as causas estruturais são "fatores de significante influência sobre o desmatamento, que seguem fortes tendências e que têm pouca probabilidade de serem instrumentos do governo para a redução do desmatamento" (COMBES MOTEL, PIRARD e COMBES, 2009, p. 385). Em outras palavras, por causas estruturais do desmatamento entendem-se aquelas que, até certo grau, não correspondem a uma variável de escolha de alguma entidade política para a redução do desmatamento.

Nesse sentido, pressupõe-se que há dois tipos de desmatamento do ponto de vista teórico. O primeiro tipo de desmatamento é o observado, correspondendo ao desmatamento que efetivamente ocorreu. $\mathrm{O}$ segundo tipo de desmatamento é o relacionado a causas estruturais, sendo esse não observado diretamente. Esse último seria a resposta padrão do desmatamento a variáveis geográficas, ambientais, sociais e econômicas não controladas pelos agentes formuladores de políticas nos estados da Amazônia Legal.

Cabe ressaltar, contudo, que várias políticas podem ser adotadas ao mesmo tempo, nas quais algumas teriam efeitos positivos sobre o desmatamento enquanto outras políticas teriam efeitos 
negativos. Nesse sentido, a diferença entre o desmatamento estrutural e o observado corresponde a um efeito líquido das políticas sobre o desmatamento em determinado período de tempo, e não o efeito parcial de alguma variável política.

Assim, poderia haver predominância de políticas de incentivo ao desmatamento, o que levaria a um efeito líquido positivo, implicando em estímulo ao desmatamento, bem como predominância de políticas de combate, que reduziriam o desmatamento. Como se pode perceber, essa abordagem se configura numa análise residual entre o desmatamento estrutural e o desmatamento observado, sendo imperativa a definição das variáveis ligadas ao desmatamento estrutural.

Combes Motel, Pirard e Combes (2009) utilizaram variáveis tanto no nível imediato quanto subjacente, como as enumeradas por Angelsen e Kaimowitz (1999), e variáveis de condições de mercado e condição inicial propostas por Silva (2009), visando identificar entre essas as que correspondem as causas estruturais do desmatamento. Dentre as variáveis de causa subjacente, são listadas como estruturais o crescimento econômico e o crescimento demográfico. Dentre as variáveis imediatas, estão a área inicial de floresta, os preços das commodities agrícolas e os custos de produção.

\section{Metodologia}

\subsection{Determinantes do desmatamento recente}

As análises desenvolvidas neste estudo consistiram de um modelo de dados em painel para modelar o desmatamento na Amazônia Legal. Baseado em Baltagi (2005) e Wooldridge (2002), esse modelo pode ser descrito na Expressão (1).

$$
D_{\mathrm{it}}=\mathrm{a}+\mathrm{a}_{\mathrm{i}}+\mathrm{b}_{\mathrm{k}} \mathrm{x}_{\mathrm{kit}}+\varepsilon_{\mathrm{it}}
$$

em que i representa as unidades de seção cruzada (estados); t são os períodos de análise, de 1999 a 2011; $D_{\text {it }}$ é o desmatamento observado no estado i no período t; a é intercepto comum a todos os estados e invariável com o tempo; $\mathrm{a}_{\mathrm{i}}$ é o efeito individual de cada estado, que capta a heterogeneidade individual por meio de um termo constante associado a um conjunto de variáveis não observadas ou latentes; $b_{k}$ é o vetor linha dos coeficientes estimados das $\mathrm{k}$ variáveis explicativas; $x_{\text {kit }}$ é o vetor coluna dessas variáveis, na unidade territorial i e período t; e $\varepsilon_{i t}$ é erro da regressão, com média zero e variância constante.

Estão representadas em $\mathrm{a}_{\mathrm{i}}$ algumas varáveis estruturais específicas de cada estado invariáveis no tempo, tais como distância do mercado consumidor e condições edafoclimáticas, além de outras condições geográficas e ambientais (PIRARD, COMBES e COMBES MOTEL, 2009). Uma característica geográfica representada no termo $\mathrm{a}_{\mathrm{i}}$ seria a importância relativa da produção de subsistência em cada estado, uma vez que tais produtos não são comercializados e, portanto, não possuem preços explícitos. Não foi considerada a estimação de dados em painel pelo modelo pooled, dado que tal especificação não controlaria as variáveis estruturais específicas de cada estado representadas pelo termo . As variáveis diretamente observadas $\left(\mathrm{x}_{\mathrm{kit}}\right)$ utilizadas foram o PIB, o PIB per capita, o PIB per capita ao quadrado, a população, a área inicial de floresta, a área relativa de floresta, a área relativa de floresta ao quadrado, o preço da soja no Brasil, o preço do boi no Brasil, índice de preços pagos pelos produtores no Brasil e os gastos do governo federal em agricultura, transportes e o crédito rural. Espera-se que o sinal dos coeficientes do PIB, PIB per capita, população, área inicial de floresta, área relativa de floresta, preço da soja, preço do boi e os gastos do governo seja positivo. Já para PIB per capita ao quadrado, área relativa de floresta ao quadrado e os preços pagos pelos produtores, sinais negativos são esperados.

Há duas formas de considerar a heterogeneidade individual $\left(\mathrm{a}_{\mathrm{i}}\right)$ apresentada na Expressão (1). A primeira consiste nos chamados Efeitos Fixos (EF), em que a heterogeneidade individual é representada pela diferença de interceptos entre as unidades de seção cruzada. Outra forma de especificar a heterogeneidade individual em relação ao desmatamento é por meio dos chama- 
098 - Desmatamento Recente nos Estados da Amazônia Legal: uma análise da contribuição dos preços agrícolas e das políticas governamentais

dos Efeitos Aleatórios (EA). Nessa abordagem, é considerado que a heterogeneidade individual é parte do erro. Retomando a Expressão (1), a estimação por EA considera $a_{i}$ um termo de erro individual, invariável no tempo e variável nas unidades de seção cruzada e $\varepsilon_{\text {it }}$ é o termo de erro variável no tempo e nas unidades de seção cruzada (BALTAGI, 2005).

Assim, há a necessidade de determinar se a estimação do painel será por EF ou EA. Wooldridge (2002) afirma que a escolha entre esses dois métodos deve levar em conta o pressuposto de $\mathrm{a}_{\mathrm{i}}$ e $\mathrm{x}_{\mathrm{kit}}$ estarem, ou não, correlacionados. Assim, se essa correlação existir, o método mais adequado é o de EF; caso contrário, utiliza-se EA, uma vez que a estimação por EF é consistente quando $a_{i}$ e $x_{\text {kit }}$ são correlacionados e a estimação por EA não o é. Para a escolha do método de estimação, realizou-se o teste proposto por Hausman (1978), que testa a ortogonalidade dos EA com os regressores. Testa-se, portanto, a hipótese nula de a estimação, por EA, ser consistente.

Outra questão econométrica importante é o problema da endogeneidade, que ocorre quando a premissa de ortogonalidade do termo de erro com as variáveis explicativas é violada na estimação por Mínimos Quadrados Ordinários (MQO). Na presença de correlação entre o termo de erro e as variáveis explicativas, os coeficientes estimados por MQO não só seriam viesados como também inconsistentes.

Dentre as variáveis utilizadas neste trabalho, a população é apontada como endógena ao processo de desmatamento por Angelsen e Kaimowitz (1999), na medida em que o processo de ocupação ocorre simultaneamente ao processo de desmatamento. O crédito rural também pode ser considerado endógeno, uma vez que a demanda por crédito rural pode estar associada ao desmatamento. Em outras palavras, o crédito rural pode financiar o desmatamento, bem como o desmatamento pode aumentar a demanda de crédito para financiar as atividades econômicas subsequentes. Assim, essas variáveis podem ser determinadas simultaneamente e, portanto, deve-se testar essa relação.
O método mais usual para identificar tal problema é o teste de Hausman para endogeneidade. Esse teste foi inicialmente proposto para identificar a endogeneidade para apenas um regressor. Wooldridge (2002) propõe um teste de endogeneidade para a possibilidade de mais de uma variável explicativa endógena. Para operacionalização do teste, é necessário que haja pelo menos uma variável instrumental associada a cada variável endógena para se construir a forma reduzida de cada uma. Apesar de não haver teoria econômica consolidada que trate dos determinantes da população e crédito rural, buscaram-se variáveis que estariam associadas a essas. No caso da população, foi utilizada como variável instrumental a densidade populacional, uma vez que se espera que, quanto maior a densidade populacional, maior seria a população. Para o crédito rural, utilizou-se o efetivo bovino e a área de lavouras como variável instrumental, esperando que haja uma relação positiva entre essas variáveis. A hipótese nula é de que os regressores não são endógenos e, para tal constatação, utiliza-se o teste de $\mathrm{F}$.

Uma vez verificada a presença de endogeneidade, os métodos de estimação por EF e EA seriam viesados e inconsistentes. Para lidar com esse problema, propõe-se a utilização de painéis dinâmicos estimados pelo Método dos Momentos Generalizados (MMG), inicialmente desenvolvido por Arellano e Bond (1991). Para esse trabalho, a Expressão (2) representa o modelo de estimação:

$$
\Delta \mathrm{D}_{\mathrm{it}}=\mathrm{e}_{1} \Delta \mathrm{D}_{\mathrm{it}-1}+\mathrm{c}_{\mathrm{m}} \Delta \mathrm{z}_{\mathrm{mit}}+\mathrm{d}_{\mathrm{n}} \Delta \mathrm{y}_{\text {nit }}+\Delta \varepsilon_{\mathrm{it}}
$$

Por meio de manipulação algébrica, o método proposto por Arellano e Bond (1991) elimina tanto a, quanto o termo individual $a_{i}$ presente na Expressão (1). Ademais, a estimação dos coeficientes é feita com as variáveis em primeira diferença ${ }^{5}$ e a variável dependente defasada é introduzida como um dos regressores. Em (2), as variáveis exógenas, representadas pelo vetor $\Delta z_{\text {mit }}$, são usa-

5. A estimação em primeira diferença evita problemas relacionados a regressões espúrias caso algumas das variáveis apresentem raiz unitária. 
das como instrumentos delas mesmas, enquanto as variáveis endógenas $\left(\Delta y_{\text {nit }}\right)$ utilizam como instrumentos as próprias variáveis defasadas em 2, $3, \ldots$ períodos (CAMERON e TRIVEDI, 2009). Para evitar a perda de graus de liberdade, foi utilizada apenas a segunda defasagem como instrumento.

Para verificar se os instrumentos utilizados são válidos, utilizou-se o teste de Sargan. Na implementação do teste, utiliza-se a estatística J de Hansen, em que, por meio do teste LM, é testada a hipótese nula de que os instrumentos utilizados são válidos (BAUM, 2006).

\subsection{Cálculo dos efeitos líquidos das políticas}

A identificação dos efeitos líquidos das políticas sobre o desmatamento nos estados foi feita por meio da análise dos resíduos da Equação (1) estimada desconsiderando-se as variáveis de políticas de crédito rural, gastos com agricultura e gastos com transporte. Esses resíduos são a diferença entre o desmatamento observado e o estrutural, ou estimado pelo modelo, e são influenciados por choques idiossincráticos nas variáveis explicativas e por variáveis aleatórias associadas a erros de medição. Diante disso, faz-se necessária a avaliação da significância estatística desse resíduo, visando anular o efeito desses elementos sobre a medição dos esforços de redução do desmatamento (COMBES MOTEL, PIRARD e COMBES, 2009; PIRARD, COMBES e COMBES MOTEL, 2009). Por meio do teste $t$ de student, foi verificado se o resíduo para cada estado em determinado período de tempo foi significativamente diferente de zero ao nível de 10\% de significância, dado o desvio padrão do resíduo.

Se o resíduo for significativo e negativo (positivo), isso indica que o desmatamento estrutural foi maior (menor) que o observado e as políticas governamentais tiveram efeitos líquidos de redução (aumento) do desmatamento no respectivo período de tempo. Se o resíduo for estatisticamente igual a zero, o desmatamento observado foi devido a características estruturais; portanto, nem políticas de incentivo, nem de mitigação do desmatamento estão sendo realizadas ou, ainda, os efeitos de políticas de incentivos foram anulados pelas políticas de redução de desmatamento da Amazônia Legal.

\subsection{Variáveis e fonte de dados}

Seguem as descrições das variáveis utilizadas neste trabalho, identificando o código, a descrição, a unidade de medida e fonte dos dados: a) desmat: área desmatada por estado da Amazônia Legal de 1999 a 2011 em km², (INPE, 2014); b) flor: área remanescente de floresta no início de cada ano, de 1999 a 2011, por estado da Amazônia Legal em km², (INPE, 2014); c) rflor: área relativa de floresta no início de cada ano (flor/ área total do estado), de 1999 a 2011, por estado da Amazônia Legal; d) rflor2: rflor elevada ao quadrado; e) psoja1: preço médio anual nacional da soja em R\$/saca defasado em um ano, (FGV, 2014); f) pboi1: preço médio anual nacional do boi gordo em R\$/arroba defasado em um ano, (FGV, 2014); g) ipp1: índice anual nacional de preços pagos pelos produtores defasados em um ano, (FGV, 2014); h) pib: produto interno bruto dos estados da Amazônia Legal de 1999 a 2011 em mil reais, (IPEA, 2014); i) ppc: produto interno bruto per capita nos estados da Amazônia Legal de 1999 a 2011 em mil reais, (IPEA, 2014); j) ppc2: ppc elevado ao quadrado; $\mathbf{k}$ ) pop: número de habitantes nos estados da Amazônia Legal de 1999 a 2011, (IPEA, 2014); 1) cr: crédito rural disponibilizado por estado da Amazônia Legal para as linhas de investimento e custeio da agricultura familiar e comercial para a agricultura e pecuária de 1999 a 2011 em mil reais, (BCB, 2014); m) ag: gastos do governo federal por estado da Amazônia Legal em mil reais com agricultura, de 1999 a 2011, relativos à promoção da produção vegetal e animal, defesa sanitária vegetal e animal, abastecimento, extensão rural, irrigação e demais gastos, (STN, 2014); n) tr: gastos do governo federal por estado da Amazônia Legal em mil reais com transportes de 1999 a 2011 relativos a investimentos no transporte rodoviário, ferroviário, hidroviário, aéreo e outros, (STN, 2014); o) lav: área de lavouras permanentes e temporárias em hectares por estado 
100 - Desmatamento Recente nos Estados da Amazônia Legal: uma análise da contribuição dos preços agrícolas e das políticas governamentais

da Amazônia Legal de 1999 a 2011 (IBGE, 2014a); p) eb: efetivo bovino em números de cabeças por estado da Amazônia legal de 1999 a 2011 (IBGE, 2014b); e q) dpop: densidade populacional é a divisão da população pela área de cada estado.

Os preços da soja, do boi e o índice de preços pagos pelos produtores são as médias nacionais de agosto do ano anterior a julho do ano corrente, período anterior às medições do Inpe para cada ano. Essas variáveis visam captar o movimento temporal dos mercados agrícolas e de insumos, uma vez que são variáveis no tempo e invariáveis no espaço. As três últimas variáveis listadas (lav, eb e dpop) foram utilizadas como variáveis instrumentais para testar a endogeneidade do crédito rural e população. As variáveis monetárias foram deflacionadas pelo IGP-DI da Fundação Getúlio Vargas (FGV) com base em dezembro de 2013. O ano de 1999 foi escolhido como o inicial pela disponibilidade de dados de crédito rural no Anuário Estatístico do Crédito Rural, que começou a ser publicado em 1999. O período de tempo foi restringido a 2011 pelo fato de não haver dados mais recentes para o PIB estadual. A análise compreende 117 observações referentes ao período de 13 anos e nove estados da Amazônia Legal.

\section{Resultados e discussão}

\subsection{Determinantes do desmatamento recente na Amazônia Legal}

As estatísticas descritivas dos dados utilizados estão na Tabela 1. Um indicador importante é o Coeficiente de Variação (CV), que mede a variabilidade de variáveis que possuem unidades de medida diferentes. $\mathrm{O}$ crédito rural, desmatamento, área de floresta, população, PIB, gastos com transporte e gastos com agricultura foram os que apresentaram maior variabilidade. Essas variáveis têm em comum o fato de serem grandezas absolutas, indicando que a variabilidade medida pelo $\mathrm{CV}$ pode estar relacionada com o tamanho do estado em questão.

As variáveis que apresentam grandezas relativas, PIB per capita e área relativa de floresta apresentaram CV de $32,33 \%$ e 53,12\%, respectivamente. Isso indica que, ao se relativizar o PIB pela população e a área de floresta pela área total, os estados da Amazônia Legal são menos heterogêneos. As variáveis de preço foram as que apresentaram menores variações em torno da média. Isso é explicado pelo fato de essas variáveis representarem apenas variações no tempo.

Tabela 1. Estatísticas descritivas para o modelo de desmatamento estadual na Amazônia Legal

\begin{tabular}{ccccccc}
\hline Variável & Unidade & Média & D. Padrão & Mín. & Máx & CV (\%) \\
\hline desmat & $\mathrm{Km}^{2}$ & 1.771 & 2.522 & 0 & 11.814 & 142,41 \\
flor & $\mathrm{Km}^{2}$ & 370.750 & 469.263 & 10.017 & 1.474 .528 & 126,57 \\
rflor & - & 0,5789 & 0,3075 & 0,0361 & 0,9447 & 53,12 \\
rflor2 & - & 0,4288 & 0,3109 & 0,0013 & 0,8925 & 72,50 \\
psoja1 & $\mathrm{R}$ /sc & 54,20 & 9,96 & 41,33 & 74,33 & 18,37 \\
pboi1 & $\mathrm{R}$ /@ & 96,02 & 8,30 & 79,43 & 105,16 & 8,64 \\
ipp1 & - & 612,83 & 28,36 & 561,41 & 665,16 & 4,63 \\
ppc & Mil R\$ & 13,51 & 4,37 & 5,69 & 26,10 & 32,33 \\
ppc2 & Mil R\$ & 201,31 & 128,46 & 32,34 & 681,39 & 63,81 \\
pib & Mil R\$ & 31.200 .000 & 24.800 .000 & 4.643 .863 & 99.300 .000 & 79,49 \\
pop & Hab & 2.578 .416 & 2.299 .683 & 266.922 & 7.688 .531 & 89,19 \\
cr & Mil R\$ & 832.387 & 1.370 .480 & 3.003 & 7.137 .252 & 164,64 \\
ag & Mil R\$ & 87.245 & 53.051 & 0 & 350.898 & 60,81 \\
tr & Mil R\$ & 335.780 & 234.227 & 27.609 & 1.191 .769 & 69,76 \\
\hline
\end{tabular}

Fonte: Resultados da pesquisa. 
Antes de se estimar o modelo, foram realizados alguns testes a fim de encontrar o método de estimação mais adequado. $\mathrm{O}$ primeiro procedimento foi o teste de Hausman para Efeitos Fixos (EF) ou Efeitos Aleatórios (EA) (Tabela 2). A hipótese nula de a estimação por EA ser consistente é rejeitada ao nível de 1\% de significância, o que mostra que o método de estimação por EF é o mais adequado para a estimação do modelo.

Após a determinação do modelo de EF para estimação do modelo do desmatamento, procedeu-se ao teste de endogeneidade de Hausman para os regressores população e crédito rural. O primeiro passo foi a estimação das equações reduzidas das duas variáveis, considerando como variáveis instrumentais a densidade populacional, para a população, e o efetivo do rebanho bovino

Tabela 2. Teste de Hausman para modelos de Efeitos Fixos ou Efeitos Aleatórios

\begin{tabular}{cc}
\hline EF $^{2}$ & Prob. EA $^{2}$ \\
\hline 30,67 & 0,000 \\
\hline
\end{tabular}

Fonte: Resultados da pesquisa. e área de lavouras permanentes e temporárias, para o crédito rural. Os resíduos das formas reduzidas de população e crédito rural foram introduzidos na estimação da forma estrutural para o desmatamento. Para finalizar, testou-se a hipótese nula de os coeficientes desses resíduos serem iguais a zero pelo teste $\mathrm{F}$ para verificar se população e crédito rural são conjuntamente endógenos. O resultado exposto na Tabela 3 indica que se rejeita a hipótese nula ao nível de $1 \%$ de significância, e, portanto, população e crédito rural são endógenos ao desmatamento.

Diante desse resultado, optou-se por estimar o modelo de desmatamento estadual por painel dinâmico proposto por Arellano e Bond (1991). Os coeficientes estimados estão na Tabela 4. A maioria dos coeficientes foi significativa a $1 \%$ e
Tabela 3. Teste de Hausman para endogeneidade nos regressores população (pop) e crédito rural (cr) para o modelo estadual

\begin{tabular}{cc}
\hline Estat. F (2;93) & P-valor $\mathbf{F}$ \\
\hline 4,95 & 0,009 \\
\hline
\end{tabular}

Fonte: Resultados da pesquisa.

Tabela 4. Coeficientes estimados para o desmatamento na Amazônia Legal

\begin{tabular}{|c|c|c|}
\hline & Coef. & Estat. Z \\
\hline l.desmat & $0,51594^{* * *}$ & 8,02 \\
\hline flor & $0,06794^{* * *}$ & 4,94 \\
\hline rflor & $57595,86^{* * *}$ & 3,45 \\
\hline rflor2 & $-44353,73^{* * *}$ & $-3,58$ \\
\hline psoja1 & $40,78^{* * *}$ & 4,57 \\
\hline pboi1 & $-35,37^{* * *}$ & $-2,97$ \\
\hline ipp1 & $-9,60^{* * *}$ & $-2,76$ \\
\hline pib & $0,00000^{\mathrm{NS}}$ & 0,08 \\
\hline ppc & $412,77^{* *}$ & 2,24 \\
\hline ppc2 & $-10,01^{*}$ & $-1,79$ \\
\hline pop & $0,00083^{\mathrm{NS}}$ & 1,11 \\
\hline $\mathrm{cr}$ & $0,00075^{* * *}$ & 3,83 \\
\hline ag & $-0,00158^{\mathrm{NS}}$ & $-0,88$ \\
\hline $\operatorname{tr}$ & $-0,00030^{\mathrm{NS}}$ & $-0,64$ \\
\hline Wald $\square^{2}$ & $\mathrm{gl}=14$ & 497,33 \\
\hline Prob. $\square^{2}$ & \multicolumn{2}{|c|}{0,0000} \\
\hline Sargan $\square^{2}$ & $\mathrm{gl}=84$ & 80,33 \\
\hline Prob. $\square^{2}$ & \multicolumn{2}{|c|}{0,5932} \\
\hline
\end{tabular}

***Significativo a $1 \%$; ** significativo a $5 \%$; * significativo a 10\%; NS não significativo a $10 \%$.

Fonte: Resultados da pesquisa. 
102 - Desmatamento Recente nos Estados da Amazônia Legal: uma análise da contribuição dos preços agrícolas e das políticas governamentais

$5 \%$, sendo a exceção o PIB, a população, os gastos com agricultura e os gastos com transporte. O teste de Wald rejeita a 1\% a hipótese nula de todos os coeficientes estimados serem iguais a zero. O teste de Sargan indica que os instrumentos utilizados para população e crédito rural são válidos.

A estimação indica que o desmatamento corrente está positivamente relacionado com o desmatamento no ano anterior, uma vez que o coeficiente da variável desmatamento defasado é significativo e positivo (l.desmat). Um aumento de $1 \mathrm{~km}^{2}$ no desmatamento em determinado estado da Amazônia Legal no ano anterior levaria a um aumento de $0,51594 \mathrm{~km}^{2}$ no ano presente. Isso indica que o desmatamento, como decisão de investimento, representa um processo contínuo ao longo do tempo. Uma decisão de investimento tomada em determinado ano terá impacto sobre os anos seguintes, mesmo que não haja mudanças nas demais variáveis que afetam o desmatamento, uma vez que tal decisão possui caráter permanente e nem todos os investimentos são concluídos no primeiro ano do projeto.

A área de florestas mostrou uma relação positiva com o desmatamento, ou seja, quanto maior a área de florestas, maior a disponibilidade de áreas a serem desmatadas. Assim, um estado com área de floresta $1 \mathrm{~km}^{2}$ maior que outro, tende a desmatar $0,068 \mathrm{~km}^{2}$ a mais. A área relativa de florestas (rflor) mostrou a relação quadrática esperada (formato de $U$ invertido). Ao se realizar a primeira derivada parcial do desmatamento com relação à área relativa de floresta e igualar essa derivada a zero, chega-se a um ponto de máximo próximo a 0,65 . Isso indica que, à direita desse ponto, o desmatamento cresce com a redução da área relativa de floresta. À esquerda desse ponto, o desmatamento se reduz mediante redução na área relativa coberta por florestas. Essa relação representa o processo de ocupação da Amazônia Legal, em que regiões com maior área relativa de florestas apresentam pequeno desmatamento inicial e, à medida que áreas são abertas por pioneiros, novos grupos de imigrantes são estimulados a ocupar o território, elevando o desma- tamento e reduzindo a área relativa de florestas. Esse aumento no desmatamento também ocorre devido à maior suscetibilidade a queimadas associada ao aumento da fragmentação florestal (NEPSTAD et al., 2008). Esse processo atinge um máximo e começa a declinar em decorrência da escassez de novas áreas a serem desmatadas. Contribui também para esse comportamento o fato de as melhores áreas para cultivo serem ocupadas nos estágios iniciais. À medida que o processo de desmatamento avança, haveria menos áreas adequadas à produção agrícola e, consequentemente, menor desmatamento.

As variáveis relacionadas ao mercado também apresentaram relação significativa com o desmatamento. Um aumento de $\mathrm{R} \$ 1,00$ no valor da saca de soja no ano anterior leva à elevação no desmatamento por estado da Amazônia Legal da ordem de $40,78 \mathrm{~km}^{2}$ no ano corrente. No caso da arroba do boi, um aumento de $\mathrm{R} \$ 1,00$ no ano anterior reduz o desmatamento atual em $35 \mathrm{~km}^{2}$. Esse último resultado é inesperado e pode estar relacionado ao fato de ter sido obtido a partir dos preços nacionais. Para os insumos utilizados na produção agrícola, um aumento de 1 ponto no índice nacional de preços pagos pelo produtor reduz o desmatamento nos estados da Amazônia Legal em 9,6 km². Diante desses resultados, foi verificada a relação do desmatamento com variáveis de mercado apresentada em outros trabalhos para a região (ARIMA et al., 2007, 2011; PFAFF, 1999; SILVA, 2009).

O PIB per capita apresentou a relação de Kuznets ambiental esperada (formato de U invertido). O ponto de máximo da relação PIB per capita com o desmatamento é de aproximadamente R\$ 20,5 mil. Assim, aumentos sucessivos no PIB per capita no intervalo de $\mathrm{R} \$ 0,00$ a $\mathrm{R} \$ 20,5 \mathrm{mil}$ aumentariam o desmatamento; após esse valor, aumentos sucessivos na renda levariam a uma redução no nível de desmatamento. Todavia, apenas Mato Grosso e Amazonas apresentaram PIB per capita acima de R\$20,5 mil em 2011, indicando que aumentos de renda nos outros estados levariam ao aumento do desmatamento. Cabe ressaltar que a evidência da Curva de Kuznets 
Ambiental para a Amazônia Legal é ambígua. Segundo Oliveira e Almeida (2010), essa relação não é observada na maioria dos municípios dessa região. Quando observada, essa relação é bastante heterogênea, apresentando diversos formatos.

O crédito rural apresentou uma relação positiva com o desmatamento, pois um aumento de R\$ 1 milhão no crédito liberado para cada estado da Amazônia Legal eleva o desmatamento em $0,75 \mathrm{~km}^{2}$. Dessa forma, constatou-se a relação esperada: a disponibilidade de crédito rural influencia a decisão de investimento dos agricultores, elevando o desmatamento. Os resultados para gastos do governo e crédito rural diferem em parte dos resultados de Prates e Serra (2009) para o estado do Pará. Esses autores chegaram a resultados semelhantes aos obtidos neste trabalho para crédito rural e gastos com agricultura. Todavia, os gastos com transporte foram significativos no estudo de Prates e Serra (2009), o que não foi verificado nesse trabalho.

\subsection{Efeitos líquidos das políticas governamentais sobre o desmatamento}

No cálculo dos efeitos líquidos não foi considerado o crédito rural, os gastos com agricultura e os gastos com transporte, pois essas são formas de política. As demais variáveis foram as mesmas do modelo apresentado na Tabela 4. A endogeneidade da população com o desmatamento não foi considerada, portanto não foi utilizado o método de estimação proposto por Arellano e Bond (1991). Os efeitos líquidos foram calculados a partir dos resíduos da estimação por efeitos fixos, seguindo Combes Motel, Pirard e Combes (2009).

Os resultados dos efeitos líquidos estão expostos na Figura 2. As linhas retas em cada diagrama representam o limite de $10 \%$ de sig- nificância, ou seja, se os resíduos ultrapassarem a linha superior (inferior) em um determinado ano, houve efeito líquido positivo (negativo) das políticas sobre o desmatamento. Os estados do Acre, Amazonas, Amapá, Maranhão, Rondônia, Roraima e Tocantins tiveram efeitos líquidos estatisticamente iguais a zero em todos os anos. Em outras palavras, o desmatamento observado foi equivalente ao desmatamento estrutural associado a variáveis não controladas pelos formuladores de políticas.

Para o estado de Mato Grosso, foram observados efeitos líquidos positivos das políticas sobre o desmatamento nos anos de 2003, 2004 e 2005, e negativos em 1999, 2000, 2009 e 2010 (Figura 2e). No Pará, o modelo indicou que houve efeitos líquidos positivos em 2004 (Figura 2f). Os estados que não apresentaram efeitos líquidos significativos não receberam políticas de desenvolvimento direcionadas para a agropecuária como parte do processo de ocupação do território Amazônico nas décadas de 1960 e 1970, conforme destacado por Lira, Silva e Pinto (2009). Com exceção do Amazonas, em que a estratégia de desenvolvimento foi a ZFM, os estados do Acre, Amapá, Maranhão, Rondônia, Roraima e Tocantins ficaram à margem do processo de desenvolvimento da região. Os estados de Mato Grosso e Pará, em que os modelos de desenvolvimento foram pautados na agropecuária, tiveram efeitos líquidos positivos em alguns anos.

Os efeitos negativos verificados nos anos de 1999 e 2000 no estado de Mato Grosso podem estar associados à mudança no código florestal realizada em 1998. Antes desse ano, o produtor rural na Amazônia Legal deveria alocar 50\% da área da propriedade para reserva legal. Devido aos altos índices de desmatamento verificados ao longo da década de 1990, o governo federal aumentou esse percentual para $80 \%$. 

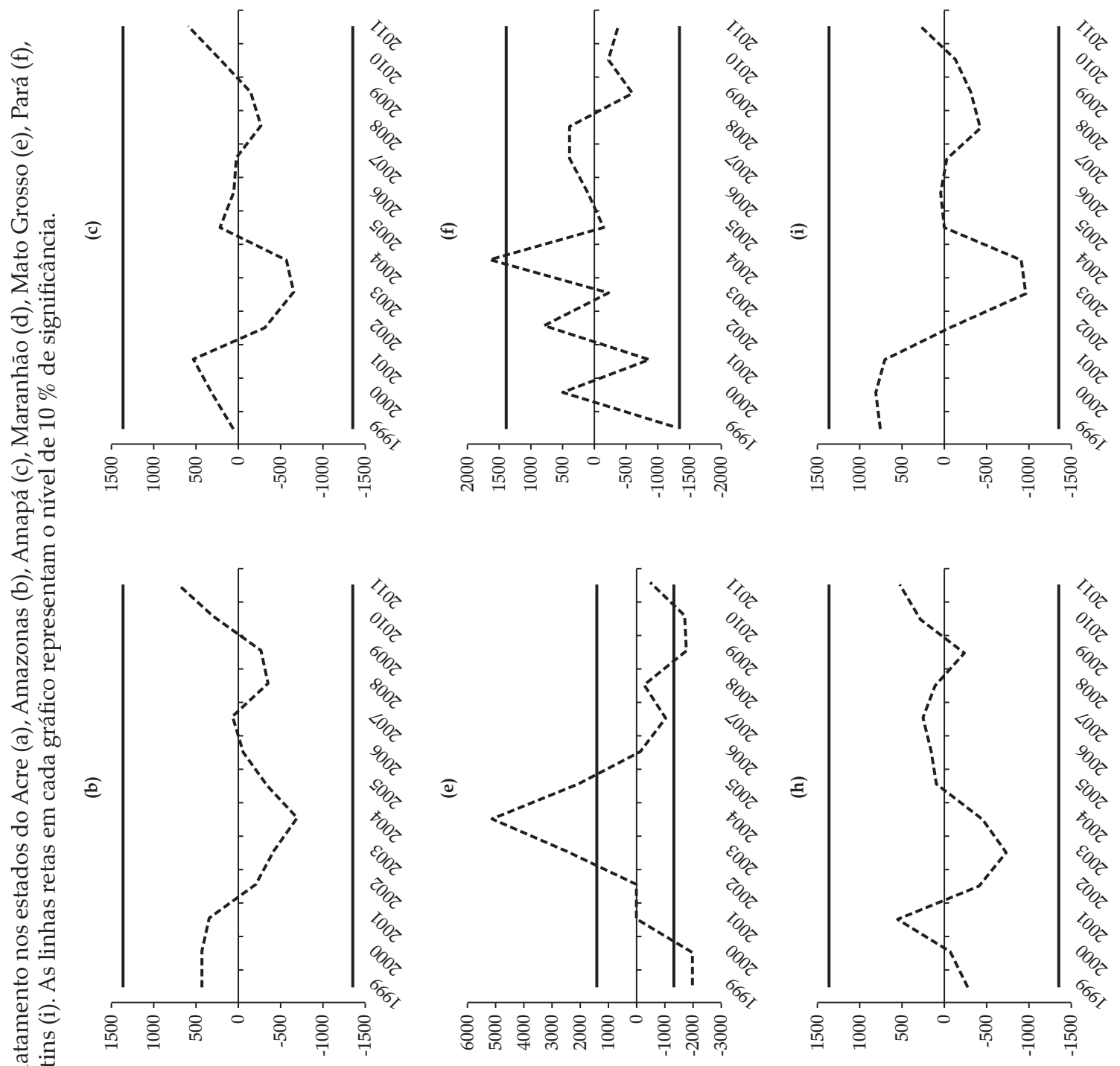

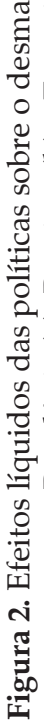
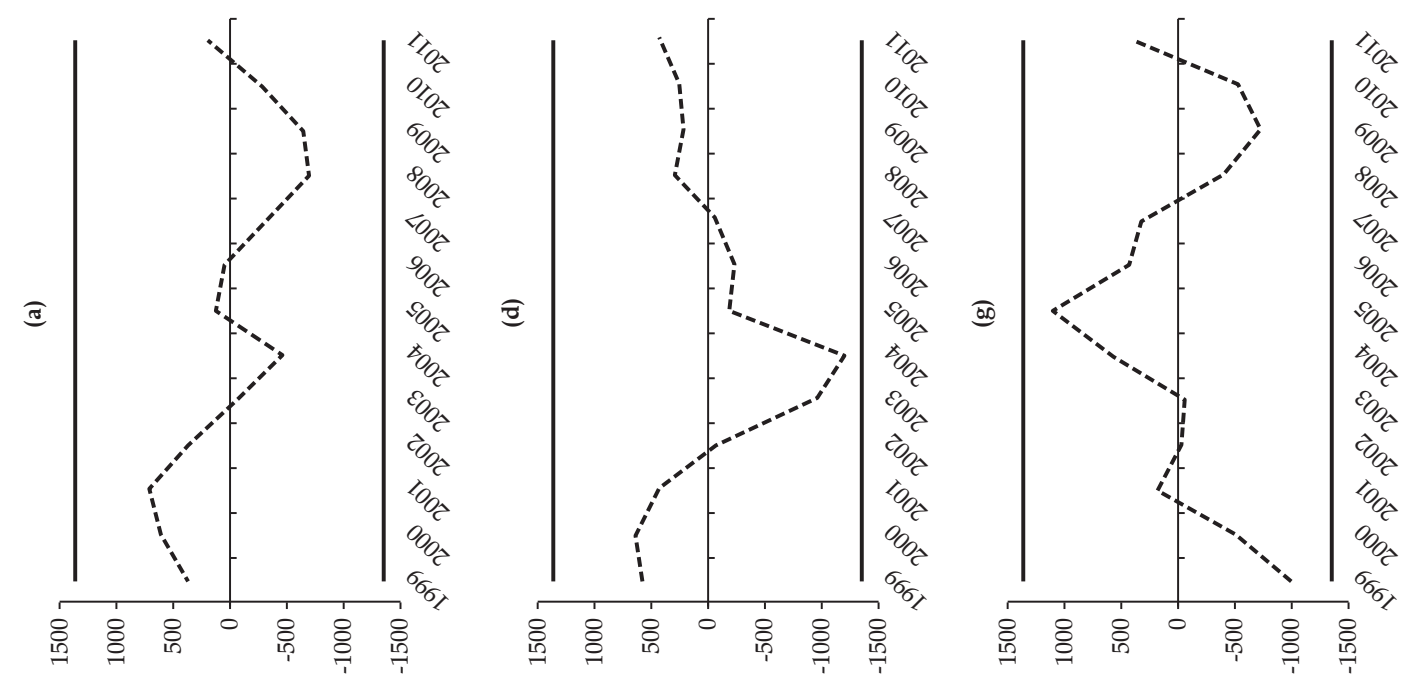

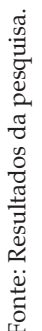


O efeito líquido das políticas em Mato Grosso seguiu tendência de queda após 2004. De 2007 em diante, o efeito líquido foi negativo e oscilou em torno da linha de significância. Apesar de o efeito de queda no desmatamento não ser significativamente diferente de zero em todos os anos no período de 2007 a 2011 em Mato Grosso, percebe-se uma mudança no comportamento da série do efeito líquido das políticas. Isso pode estar relacionado com as políticas de redução do desmatamento implantadas pelo governo federal a partir de 2004 e intensificadas em 2008, conforme Barreto e Silva (2010). Outros trabalhos também chegaram a resultados semelhantes. Assunção, Gandour e Rocha (2012) indicam que as políticas aplicadas a partir de 2004 e intensificadas em 2008 reduziram o desmatamento em mais de 60.000 $\mathrm{km}^{2}$ de 2005 a 2009. Assunção, Gandour e Rocha (2013) chegaram a um número próximo para o efeito das políticas de fiscalização e aplicação de multas de 2007 a 2011. Embora com caráter qualitativo, os resultados obtidos neste trabalho complementam os de Assunção, Gandour e Rocha (2012) e Assunção, Gandour e Rocha (2013)

\section{Considerações finais}

Foi constatado neste trabalho que houve preponderância das variáveis estruturais, principalmente preços agrícolas na explicação do desmatamento. Os gastos do governo federal relacionados a políticas de estímulo não tiveram grande influência no desmatamento. No caso do crédito rural, essa relação se mostrou significativa do ponto de vista estatístico.

O relacionamento mais expressivo do desmatamento com o preço da soja e dos insumos agrícolas parece indicar que a qualquer momento pode haver uma reversão do processo de redução do desmatamento observado recentemente, desde que as condições sejam favoráveis a isso (alta de preços da soja, queda de custos agrícolas etc..). Entretanto, os efeitos líquidos negativos em Mato Grosso observados nos últimos anos indicam maior efetividade de políticas públicas, o que também foi verificado em outros trabalhos recentes.

Foi confirmada a presença de endogeneidade do crédito rural e da população em relação ao desmatamento, reforçando, assim, a necessidade do tratamento correto dessas variáveis na modelagem do desmatamento no nível de estados da Amazônia Legal em trabalhos futuros.

Os resultados deste trabalho indicam que as políticas públicas possuem papel relevante sobre o desmatamento na Amazônia Legal. Como avaliação de tais políticas, o presente estudo sugere que as políticas de comando e controle implementadas a partir de 2004 foram efetivas na redução do desmatamento, principalmente em Mato Grosso. Nesse sentido, tais políticas deveriam ser mantidas. De acordo com as estimações, tais políticas deveriam ser intensificadas em momentos em que o preço da soja esteja maior, uma vez que esse preço mostrou-se relevante na explicação do desmatamento. Todavia, a participação da agricultura de subsistência como determinante do desmatamento não foi avaliada nesse trabalho. Tal categoria poderia ter papel relevante nesse contexto, principalmente pelo fato de não responder às variáveis de mercado, como a agricultura comercial. Assim, generalizações a partir dos resultados deste trabalho não abarcariam o comportamento da agricultura de subsistência.

\section{Referências bibliográficas}

ANDERSEN, L. E. et al. The dynamics of deforestation and economic growth in the Brazilian Amazon. Cambridge: Cambridge University Press, 2002. p. 259

ANGELSEN, A. Agricultural expansion and deforestation: modelling the impact of population, market forces and property rights. Journal of Development Economics, v. 58, n. 1, p. 185-218, fev. 1999.

. e KAIMOWITZ, D. Rethinking the causes of deforestation: Lessons from economic models. The World Bank Research Observer, v. 14, n. 1, p. 73-98, fev. 1999.

ARAUJO, C. et al. Property rights and deforestation in the Brazilian Amazon. Ecological Economics, v. 68, n. 8-9, p. 2461-2468, jun. 2009. 
106 - Desmatamento Recente nos Estados da Amazônia Legal: uma análise da contribuição dos preços agrícolas e das políticas governamentais

ARELLANO, M. e BOND, S. Some tests of specification for panel data: Monte Carlo evidence and an application to employment equations. The Review of Economic Studies, v. 58, n. 2, p. 277-297, 1991.

ARIMA, E. Y. et al. Fire in the Brazilian Amazon: A spatially explicit model for policy impact analysis. Journal of Regional Science, v. 47, n. 3, p. 541-567, ago. 2007.

. et al. Statistical confirmation of indirect land use change in the Brazilian Amazon. Environmental Research Letters, v. 6, n. 2, p. 024010, 1 abr. 2011.

ASSUNÇÃO, J., GANDOUR, C. C. e ROCHA, R. Deforestation slowdown in the Legal Amazon: prices or policies?: CPI. Rio de Janeiro: [s.n.]. 2012. Disponível em: <http://climatepolicyinitiative.org/wp-content/ uploads/2012/03/Deforestation-Prices-or-PoliciesWorking-Paper.pdf $>$.

., GANDOUR, C. e ROCHA, R. DETERing deforestation in the Brazilian Amazon: Environmental monitoring and law enforcement. Rio de Janeiro: [s.n.]. 2013 Disponível em: <http://www.econ.puc-rio.br/ uploads/adm/trabalhos/files/Command_and_Control. pdf $>$.

BALTAGI, B. Econometric analysis of panel data. 3. ed. Chichester: JohnWiley \& Sons Ltd, 2005. p. 302.

BARRETO, P., PEREIRA, R. e ARIMA, E. A pecuária e o desmatamento na Amazônia na era das mudanças climáticas. Belém: Instituto do Homem e Meio Ambiente da Amazônia, 2008. p. 42

. e SILVA, D. Will cattle ranching continue to drive deforestation in the Brazilian Amazon. Belém: [s.n.]. Disponível em: <http://www.imazon.org.br/ publicacoes/congressos-e-anais/will-cattle-ranchingcontinue-to-drive >. Acesso em: 10 dez. 2013.

BAUM, C. F. An introduction to modern econometrics using Stata. 1. ed. College Station, Texas: Stata Press, 2006. p. 343.

BCB - BANCO CENTRAL DO BRASIL. Anuário Estatístico do Crédito Rural. Disponível em: <http:// www.bcb.gov.br/?CREDRURAL > . Acesso em: 25 ago. 2014.

BRASIL - MINISTÉRIO DA CIÊNCIA E TECNOLOGIA. Estimativas anuais de emissões de gases de efeito estufa no Brasil. Brasília, DF: [s.n.]. Disponível em: < http://www. mcti.gov.br $>$.

CAMERON, A. C. e TRIVEDI, P. K. Microeconometrics Using Stata. 1. ed. College Station, Texas: Stata Press, 2009. p. 692
CAMMELLI, F. Smallholders' collective action and fire risk in the Brazilian Amazon. 133 f. Dissertação (Mestrado em Desenvolvimento Econômico) - Scuola di Economia e Management, Università Degli Studi Firenze, Firenze. 2013.

CAMPARI, J. S. The Economics of Deforestation in the Amazon. 1. ed. Cheltenham: Edward Elgar Publishing, 2005. p. 242

COHN, A. S. et al. Cattle ranching intensification in Brazil can reduce global greenhouse gas emissions by sparing land from deforestation. Proceedings of the National Academy of Sciences of the United States of America, v. 111, n. 20, p. 7236-41, 20 maio 2014.

COMBES MOTEL, P., PIRARD, R. e COMBES, J. A methodology to estimate impacts of domestic policies on deforestation: Compensated Successful Efforts for "avoided deforestation" (REDD). Ecological Economics, v. 68 , n. 3, p. 680-691, jan. 2009.

DE SOUZA, R. A., MIZIARA, F. e DE MARCO JUNIOR, P. Spatial variation of deforestation rates in the Brazilian Amazon: A complex theater for agrarian technology, agrarian structure and governance by surveillance. Land Use Policy, v. 30, n. 1, p. 915-924, jan. 2013.

DEFRIES, R. et al. Export-oriented deforestation in Mato Grosso: harbinger or exception for other tropical forests? Phil. Trans. R. Soc. B, v. 368, n. 1619, p. 1-8, 2013.

DINIZ, M. B. et al. Causas do desmatamento da Amazônia: uma aplicação do teste de causalidade de Granger acerca da Amazônia Legal brasileira. Nova Economia, v. 19, n. 1, p. 121-151, 2009.

FEARNSIDE, P. M. Deforestation in Brazilian Amazonia: history, rates, and consequences. Conservation Biology, v. 19 , n. 3, p. 680-688, jun. 2005.

FÉRES, J. G. e ARAUJO, C. Determinants of Amazon deforestation: the role of off-farm income. LI Congresso da Sober. Anais... Belém: Sober, 2013.

FGV - FUNDAÇÃO GETÚLIO VARGAS. FGVdados. Disponível em: < http://portalibre.fgv.br>. Acesso em: 25 ago. 2014.

FOOD AND AGRICULTURE ORGANIZATION OF THE UNITED NATIONS. Global Forest Resources Assessment 2010: Main Report. Roma, Itália: FAO, 2010. Disponível em: <http://www.fao.org/docrep/013/ i1757e/i1757e.pdf>.

GALFORD, G. L., SOARES-FILHO, B. e CERRI, C. E. P. Prospects for land-use sustainability on the agricultural frontier of the Brazilian Amazon. Phil. Trans. R. Soc. B, v. 368 , n. 1619, p. 1-5, 2013. 
GEIST, H. J. e LAMBIN, E. F. Proximate causes and underlying driving forces of tropical deforestation. BioScience, v. 52, n. 2, p. 143, 2002.

HAUSMAN, J. A. Specification tests in econometrics. Econometrica, v. 46, p. 1251-1272, 1978.

HECHT, S. B. Environment, development and politics: capital accumulation and the livestock sector in Eastern Amazonia. World Development, v. 13, n. 6, p. 663-684, jun. 1985.

HOMMA, A. K. O. Expansão agropecuária e desmatamento na Amazônia: Quais os caminhos. In: COELHO, A. B., TEIXEIRA, E. C. e BRAGA, M. J. (Eds.). Recursos Naturais e Crescimento Econômico. 1. ed. Viçosa, MG: [s.n.]. p. 125-176.

IBGE - INSTITUTO BRASILEIRO DE GEOGRAFIA E ESTATÍSTICA. Indicadores de Desenvolvimento Sustentável: Brasil 2010. Rio de Janeiro, RJ: [s.n.]. Disponível em: <http://www.ibge.gov.br/home/ geociencias/recursosnaturais/ids/ids2010.pdf $>$.

IBGE - INSTITUTO BRASILEIRO DE GEOGRAFIA E ESTATÍSTICA. Pesquisa Agrícola Municipal. Disponível em: < http://www.sidra.ibge.gov.br>. Acesso em: 25 ago. 2014a.

IBGE - INSTITUTO BRASILEIRO DE GEOGRAFIA E ESTATÍSTICA. Pesquisa Pecuária Municipal. Disponível em: < http://www.sidra.ibge.gov.br>. Acesso em: 25 ago. 2014b.

INPE - INSTITURO NACIONAL DE PESQUISAS ESPACIAIS. Projeto Prodes - Monitoramento da floresta amazônica brasileira por satélite. Disponível em: <http:// www.obt.inpe.br/prodes/index.php>. Acesso em: 19 mar. 2014.

IPEA - INSTITUTO DE PESQUISA ECONÔMICA APLICADA. Dados Macroeconômicos e Regionais. Disponível em: < http://www.ipeadata.gov.br/ipeaweb. dll/ipeadata?991206562 >. Acesso em: 25 ago. 2014.

LIRA, S. R. B. D., SILVA, M. L. M. e PINTO, R. S. Desigualdade e heterogeneidade no desenvolvimento da Amazônia no século XXI. Nova Economia, v. 19, n. 1, p. 153-184, 2009.

MARCHAND, S. The relationship between technical efficiency in agriculture and deforestation in the Brazilian Amazon. Ecological Economics, v. 77, p. 166175, maio 2012.

NEPSTAD, D. et al. Slowing Amazon deforestation through public policy and interventions in beef and soy supply chains. Science, v. 344, n. 6188, p. 1118-23, 6 jun. 2014.
. et al. Interactions among Amazon land use, forests and climate: prospects for a near-term forest tipping point. Phil. Trans. R. Soc. B, v. 363, n. 1498, p. 1737-46, 27 maio 2008.

OLIVEIRA, R. C. e ALMEIDA, E. Desmatamento da Amazônia Legal e heterogeneidade espacial: uma abordagem local para a curva de Kuznets ambiental. VIII Encontro Nacional da Associação de Estudos Regionais e Urbanos. Anais... Juiz de Fora: Aber, 2010. Disponível em: < http://www.estudosregionais.org.br/ admin/upload/File/A147.pdf $>$

PFAFF, A. S. P. What drives deforestation in the Brazilian Amazon? Journal of Environmental Economics and Management, v. 37, n. 1, p. 26-43, jan. 1999.

PIRARD, R., COMBES, J. e COMBES MOTEL, P. A response to the commentary on "Compensated Successful Efforts." Ecological Economics, v. 68, n. 8-9, p. 2179-2181, jun. 2009.

PRATES, R. C. e SERRA, M. O impacto dos gastos do governo federal no desmatamento no Estado do Pará. Nova Economia, v. 19, n. 1, p. 95-116, 2009.

SANT'ANNA, A. A. e YOUNG, C. E. F. Direitos de propriedade, desmatamento e conflitos rurais na Amazônia. Economia Aplicada, v. 14, n. 3, p. 381-393, 2010.

SILVA, J. H. G. Economic causes of deforestation in the Brazilian Amazon: an empirical analysis of the 2000s. XXXVII ENCONTRO NACIONAL DE ECONOMIA. Anais... Foz do Iguaçu: Anpec, 2009. Disponível em: $<$ http://www.anpec.org.br/encontro_2009.htm>. Acesso em: 9 dez. 2013

SOUZA-RODRIGUES, E. A. Demand for Deforestation in the Amazon. Cambridge, MA: [s.n.]. 2012. Disponível em: <http://environment.harvard.edu/events/2012-09-25/ demand-deforestation-amazon $>$.

STN - SECRETARIA DO TESOURO NACIONAL. Estados e Municípios. Disponível em: <http://www. stn.fazenda.gov.br/estados_municipios/index.asp $>$. Acesso em: 25 ago. 2014.

SUDAM - SUPERINTENDÊNCIA DO DESENVOLVIMENTO DA AMAZÔNIA. Legislação sobre a criação da Amazônia Legal. Disponível em: <http://www.e.gov.br/defaultCab. asp?idservinfo $=35616 \& u r l=h t t p: / / w w w \cdot a d a \cdot g o v \cdot b r /$ index.php?option $=$ com_content\&task $=$ category\&secti onid $=9 \& \mathrm{id}=54 \&$ Itemid $=51>$. Acesso em: 20 ago. 2010 .

WALKER, R., MORAN, E. e ANSELIN, L. Deforestation and cattle ranching in the Brazilian Amazon: external capital and household processes. World Development, v. 28, n. 4 , p. 683-699, abr. 2000. 
108 - Desmatamento Recente nos Estados da Amazônia Legal: uma análise da contribuição dos preços agrícolas e das políticas governamentais

WEINHOLD, D. e REIS, E. Transportation costs and the spatial distribution of land use in the Brazilian Amazon. Global Environmental Change, v. 18, n. 1, p. 54-68, fev. 2008.
WOOLDRIDGE, J. M. Econometric Analysis of Cross Section and Panel Data. 1. ed. Cambridge, MA: MIT Press, 2002. v. 58p. 752. 